\title{
Cross-Linked Polymer Hydrogel Electrolytes for Electrochemical Capacitors
}

\author{
N. A. Choudhury, ${ }^{a}$ A. K. Shukla, ${ }^{a, c, *, z}$ S. Sampath, ${ }^{\text {b }}$ and S. Pitchumani ${ }^{c}$ \\ ${ }^{a}$ Solid State and Structural Chemistry Unit, Indian Institute of Science, Bangalore-560 012, India \\ ${ }^{b}$ Inorganic and Physical Chemistry Department, Indian Institute of Science, Bangalore-560 012, India \\ ${ }^{c}$ Central Electrochemical Research Institute, Karaikudi-630 006, India
}

Acidic polyvinyl alcohol/polyacrylic acid blend hydrogel electrolytes (BHEs) were prepared by cross-linking with glutaraldehyde and perchloric acid. These acidic BHEs were treated suitably to realize alkaline and neutral BHEs. Amorphicity of BHEs was followed by differential scanning calorimetry. Ionic conduction in acidic BHEs was found to take place by a Grötthus-type mechanism whereas in alkaline and neutral BHEs it was due to a segmental motion mechanism. Ionic conductivity of BHEs was found to range between $10^{-3}$ and $10^{-2} \mathrm{~S} \mathrm{~cm}^{-1}$. Electrochemical capacitors assembled with acidic polyvinyl alcohol hydrogel electrolyte yielded a maximum capacitance of $\sim 60$ and $1000 \mathrm{~F} \mathrm{~g}^{-1}$ with $\mathrm{BP}$ carbon and $\mathrm{RuO}_{x} \cdot x \mathrm{H}_{2} \mathrm{O} / \mathrm{C}$ electrodes, respectively. (c) 2006 The Electrochemical Society. [DOI: 10.1149/1.2164810] All rights reserved.

Manuscript submitted September 2, 2005; revised manuscript received December 2, 2005.

Available electronically February 1, 2006

Polymer electrolytes, such as polyethylene oxide-salt complexes, ${ }^{1-4}$ are the most common solid electrolytes employed in electrochemical devices. Solid polymer electrolytes have ionic conductivity between $10^{-8}$ and $10^{-7} \mathrm{~S} \mathrm{~cm}^{-1}$, which are too low to be of use in practical devices. Efforts ${ }^{1}$ have, therefore, been expended to enhance the ionic conductivity of polymer electrolytes. One such approach involves addition of a low molecular-weight polar solvent, called plasticizer, to a polymer-salt system to form a polymer gel electrolyte (PGE) ${ }^{5-8}$ PGEs have good adhesive properties, liquidlike behavior, and exhibit high ionic conductivity of $\sim 10^{-3} \mathrm{~S} \mathrm{~cm}^{-1}$ at ambient temperatures. Although nonaqueous PGEs have a wider potential window of $\sim 4 \mathrm{~V}$ as compared to $\sim 1 \mathrm{~V}$ for aqueous PGEs, handling of the former require a moisture-free environment which is both tedious and costly to maintain. Moreover, organic solvents used as plasticizers with nonaqueous PGEs are environmentally malignant. $^{5}$

Electrochemical capacitors (ECs) are electrical devices with highly reversible charge-storage and delivery capabilities. ECs have properties complementary to secondary batteries and find usage in hybrid power systems for electric vehicles, heavy-load starting assist for diesel locomotives, utility load leveling, military, and medical applications. ${ }^{9}$ ECs employ both aqueous and nonaqueous electrolytes in either liquid or solid state. Solid electrolytes provide the advantages of compactness and reliability without leakage of liquid components. Depending on the charge-storage mechanism, an EC is classified as an electrical double layer capacitor (EDLC) or a pseudocapacitor. Higher energy density of EDLCs, as compared to dielectric capacitors, is mainly due to the large surface area of the electrode materials, usually comprising activated carbons, ${ }^{10,11}$ aerogel or xerogel carbons, ${ }^{10,12-14}$ and carbon nanotubes. ${ }^{10,15,16}$ Doublelayer capacitors have several advantages over secondary batteries, such as faster charge-discharge, longer cycle-life ( $>10^{5}$ cycles), and higher power density. ${ }^{9}$ Pseudocapacitors are also called redox capacitors because of the involvement of redox reactions in the charge-storage and delivery processes. Energy storage mechanisms in pseudocapacitors involve fast faradaic reactions such as underpotential deposition, intercalation or redox process occurring at or near a solid electrode surface at an appropriate potential. ${ }^{9}$ Redox processes often occur in conducting polymers ${ }^{9,17}$ and metal oxides, ${ }^{9}, 18-27$ making them attractive materials for pseudocapacitors.

Reportedly, organic PGEs have been used in both lithium batteries $^{5}$ and electrochemical double-layer capacitors (EDLC). ${ }^{6-8}$ Also, there have been reports ${ }^{28,29}$ of aqueous PGEs based on PVA- $\mathrm{H}_{3} \mathrm{PO}_{4}$ and their use in electrochemical capacitors. But, owing to their corrosive nature, acidic electrolytes are suitable only to a

\footnotetext{
* Electrochemical Society Active Member.

z E-mail: shukla@sscu.iisc.ernet.in
}

limited number of electrode materials. Alkaline PGEs, such as PEO-KOH- $\mathrm{H}_{2} \mathrm{O}^{30-32}$ and PAA-KOH- $\mathrm{H}_{2} \mathrm{O},{ }^{33,34}$ have found applications in nickel-metal hydride batteries and EDLCs. However, an aqueous PGE suitable to acidic, alkaline and neutral environments is lacking in the literature. Blending of two or more polymers is an interesting route to prepare a polymeric material with properties better than those of the individual component polymers. ${ }^{35-37}$

In light of the aforesaid discussion, we have chosen a polyvinyl alcohol/polyacrylic acid (PVA/PAA) blend to realize an aqueous PGE amenable to acidic, alkaline, and neutral environments. PVA/ PAA blends are known to have film integrity and good mechanical properties, which can be enhanced by chemical cross-linking with glutaraldehyde. These films have high water retention capacity and hence are pliable for fine-tuning their ionic conductivity. PVA/PAA blends have been reported as modified electrodes for application in electrochemical sensors ${ }^{38}$ as well as in ion-exchange polymers. ${ }^{39}$

In this study, we report preparation with characterization of polymer hydrogel electrolytes comprising glutaraldehyde cross-linked PVA/PAA blends with $\mathrm{HClO}_{4}, \mathrm{NaOH}$, and $\mathrm{NaCl}$ dopants as well as their use in electrochemical capacitors. Among these, the EC with acidic pristine PVA hydrogel electrolyte and carbon-supported $\mathrm{RuO}_{x} \cdot x \mathrm{H}_{2} \mathrm{O}$ electrodes exhibited a capacitance value as high as $1000 \mathrm{~F} \mathrm{~g}^{-1}$.

\section{Experimental}

Preparation of acidic PVA/PAA blend hydrogel electrolyte.Acidic polyvinyl alcohol/polyacrylic acid BHEs were prepared by a modified solution casting method ${ }^{38}$ in which varied volume ratios of aq. PVA $(\mathrm{Mw} \approx 14000$, BDH Ltd., England $)$ and aq. PAA (63 wt $\%$ aq. solution, $d=1.23 \mathrm{~g} / \mathrm{cm}^{3}$, Acros Organics, USA) were mixed with optimized amounts of glutaraldehyde (50\% aq. solution, Merck Schuchardt OHG, Germany), and perchloric acid $\left(\sim 70 \% ; d=1.67 \mathrm{~kg} \mathrm{~L}^{-1}\right.$, Merck Ltd., India). In a typical preparation, required volumes of $10 \%(\mathrm{w} / \mathrm{v})$ aq. solutions of each of PVA and PAA were mixed at room temperature $(\sim 298 \mathrm{~K})$ using a magnetic stirrer for $\sim 3 \mathrm{~h}$, with the total volume of PVA and PAA solutions in each hydrogel being maintained constant at $5.0 \mathrm{~mL}$. To this admixture of polymer solutions, $0.5 \mathrm{~mL}$ of $5 \%$ aq. glutaraldehyde was added dropwise with stirring continued for $\sim 2 \mathrm{~h}$. Subsequently, $50 \mu \mathrm{L}$ of $1.5 \mathrm{M}$ perchloric acid $\left(\mathrm{HClO}_{4}\right)$ was added dropwise to catalyze the cross-linking reaction between PVA and glutaraldehyde with stirring continued till the onset of gelation when the contents were transferred to a Petri dish and kept at room temperature for the gelation to complete. Since the gelation involved reaction between PVA and glutaraldehyde, the time required for the gelation depended on the proportion of PVA in the blend. In a typical preparation, the 
gelation took $\sim 10 \mathrm{~min}, \sim 30 \mathrm{~min}$ and $\sim 1 \mathrm{~h}$, respectively, for the hydrogels with PVA: PAA compositions of 5:0 (pristine PVA), 4:1 and $3: 2$.

Preparation of alkaline PVA/PAA blend hydrogel electrolyte.Alkaline polyvinyl alcohol/polyacrylate salt BHE was prepared by neutralization of the as-prepared acidic BHE with an alkali metal hydroxide followed by treatment with the appropriate alkaline electrolyte solution. For the preparation of BHE with $\mathrm{NaOH}$ dopant, the acidic BHE of $\sim 1 \mathrm{~cm}^{2}$ area was first neutralized by treating with $5 \mathrm{~mL}$ of $5 \mathrm{M} \mathrm{NaOH}$ at $313-323 \mathrm{~K}$ for $\sim 3 \mathrm{~h}$. During this process, the transparent BHE changed from colourless to yellow. After complete neutralization, the BHE was washed repeatedly with hot millipore water $(\sim 333 \mathrm{~K})$ until it was found to be devoid of ionic species and ionically nonconducting, as verified by electrochemical impedance spectroscopy. This blend hydrogel was then kept immersed in $5 \mathrm{~mL}$ of $0.5 \mathrm{M} \mathrm{NaOH}$ solution for $\sim 12 \mathrm{~h}$ at room temperature in order to attain electrolyte saturation in the hydrogel matrix.

Preparation of neutral PVA/PAA blend hydrogel electrolyte.Neutral PVA/PAA BHE was prepared in a similar way as that of the alkaline BHE except for the last step wherein the nonconducting hydrogel matrix of $\sim 1 \mathrm{~cm}^{2}$ area, obtained after neutralization of the as-prepared acidic BHE with alkali followed by repeated washing, was treated with $5 \mathrm{~mL}$ of the appropriate salt solution, e.g., $0.5 \mathrm{M}$ $\mathrm{NaCl}$, instead of the alkali.

Preparation of amorphous hydrated ruthenium oxide-carbon composite. - A sol-gel route $\mathrm{r}^{20,40}$ was followed to prepare $10 \%$ (w/w) carbon-supported amorphous hydrated ruthenium oxide $\left(\mathrm{RuO}_{x} \cdot x \mathrm{H}_{2} \mathrm{O} / \mathrm{C}\right)$. Required amount of $\mathrm{BP}$ carbon was suspended in millipore water and agitated in an ultrasonic water bath to form slurry. To the resultant slurry, required amount of $\mathrm{RuCl}_{3} \cdot \mathrm{xH}_{2} \mathrm{O}$ dissolved in aq. methanol (1:1) was added dropwise with constant stirring. During this process, the $\mathrm{pH}$ was maintained constant at $\sim 7$ by adding $0.3 \mathrm{M} \mathrm{NaOH}$. The black colored $\mathrm{RuO}_{x} \cdot x \mathrm{H}_{2} \mathrm{O} / \mathrm{C}$ was filtered, washed copiously with hot Millipore water, and dried in a hot air oven at $\sim 338 \mathrm{~K}$ for $6 \mathrm{~h}$.

Physical characterization of ruthenium oxide-carbon composites.-Ruthenium oxide-carbon composites were characterized by recording their powder X-ray diffraction (XRD) patterns on a Siemens D-5005 X-ray diffractometer using $\mathrm{Cu} \mathrm{K} \alpha$ radiation.

Thermal characterization of PVA/PAA blend hydrogel electrolyte.-Acidic PVA/PAA BHEs of varied polymer compositions were characterized ${ }^{28,41}$ by differential scanning calorimetry using a Mettler Toledo MET_E-3561 DSC 822e differential scanning calorimeter.

Electrochemical characterization of PVA/PAA blend hydrogel electrolytes. - Electrochemical characterizations of acidic, alkaline and neutral PVA/PAA BHEs were carried out by studying their ionic conductivity and capacitor performances. For ionic conductivity measurements, the BHEs were sandwiched between two symmetrical blocking stainless steel (SS) electrodes, each of $\sim 0.5 \mathrm{~cm}^{2}$ in geometrical area. For capacitor performance studies, all solid-state $\mathrm{ECs}^{42,43}$ were assembled with various hydrogel electrode assemblies (HEA). The HEAs comprised optimized PVA/PAA BHEs with acidic, alkaline, and neutral dopants, sandwiched between two carbon papers (Toray TGP-H-090) on which the electrode materials were coated. The electrodes used included BP carbon and asprepared $10 \%(\mathrm{w} / \mathrm{w}) \mathrm{RuO}_{x} \cdot x \mathrm{H}_{2} \mathrm{O} / \mathrm{C}$. To prepare electrodes for ECs, a slurry was obtained by ultrasonicating the required amount of the electrode material with $40 \%(w / w)$ Nafion perfluorinated ionexchange resin (5 wt \% solution, Aldrich Chemical Company, Inc., USA) in iso-propyl alcohol. The slurry was dropped on to a preweighed BP carbon-coated carbon paper (Toray TGP-H-090) of $0.28 \mathrm{~mm}$ thickness and $1 \mathrm{~cm}^{2}$ geometrical area. The loading of the electrode material was $\sim 0.1 \mathrm{mg} \mathrm{cm}^{-2}$, which was kept identical for all the ECs studied here. Electrodes of the HEAs were contacted on their rear with SS current collectors of $0.15 \mathrm{~mm}$ thickness and $1 \mathrm{~cm}^{2}$ geometrical area. The HEAs with the SS current collectors were fixed inside a fixture comprising two circular Teflon disks with SS screws. After installing the single EC cells in the test station, performance evaluation studies were initiated.

Electrochemical characterization of the BHEs were carried out by cyclic voltammetry (CV), electrochemical impedance spectroscopy (EIS) and galvanostatic charge-discharge studies. All the electrochemical studies were carried out using an Autolab Electrochemical System (Eco Chemie BV, The Netherlands). All the electrochemical measurements except the temperature dependence of ionic conductivity were conducted at room temperature.

The bulk ionic conductivity $(\sigma)$ of the hydrogel electrolytes were determined from the complex impedance spectra in the frequency range between $200 \mathrm{~Hz}$ and $10 \mathrm{kHz}$ with a perturbation of $5 \mathrm{mV} \mathrm{rms}$ using the equation

$$
\sigma=L / R A
$$

In Eq. 1, $L, A$, and $R$ are, respectively, the thickness, area, and bulk resistance (calculated from high-frequency intercept on the real impedance axis of the Cole-Cole plot) of the PVA/PAA BHE.

To evaluate utility of the various BHEs in ECs, CVs were recorded in the potential range between -0.5 and $+0.5 \mathrm{~V}$ for acidic BHE with BP carbon electrodes, and between -0.2 and $+0.8 \mathrm{~V}$ for acidic BHE with $\mathrm{RuO}_{x} \cdot x \mathrm{H}_{2} \mathrm{O} / \mathrm{C}$ electrodes, between -0.4 and $+0.4 \mathrm{~V}$ for alkaline BHE with BP carbon electrodes, and between -0.3 and $+0.3 \mathrm{~V}$ for neutral BHE with BP carbon electrodes using a two-electrode configuration. All the $\mathrm{CVs}$ were recorded at a scan rate of $2 \mathrm{mV} \mathrm{s}^{-1}$. The specific capacitance (C) values were obtained from the CVs using the equation

$$
C=I /[w(d E / d t)]
$$

In Eq. $2, I$ is the current, $w$ is the total weight of the electrode material used in the two electrodes and $d E / d t$ is the potential scan rate.

The charge-transfer resistance, phase angle and relaxation time constant $\left(\tau_{o}\right)$ of the ECs were determined from the complex impedance spectra recorded in the frequency range between $5 \mathrm{mHz}$ and $10 \mathrm{kHz}$ with a perturbation of $5 \mathrm{mV}$ rms.

Life-cycle tests were performed with galvanostatic chargedischarge measurements. The specific capacitance of the ECs was calculated from the discharge curves of the charge-discharge plots using the equation

$$
C=I \Delta t /[w \Delta V]
$$

In Eq. 3, $I$ is the constant current and $\Delta t$ is the time required to change the potential by $\Delta \mathrm{V}$.

\section{Results and Discussion}

The cross-linking reaction between PVA blended with PAA and glutaraldehyde leading to the formation of acidic PVA/PAA BHE and its subsequent neutralization with alkali is schematically depicted in Fig. 1. The as-prepared acidic PVA/PAA BHEs were $\sim 1.5 \mathrm{~mm}$ in thickness, transparent, colorless, and mechanically stable with abundant amount of water absorbed in the polymer hydrogel matrix. Its ionic conductivity $\left(\sim 10^{-3} \mathrm{~S} \mathrm{~cm}^{-1}\right)$ was mostly due to $\mathrm{HClO}_{4}$, albeit the weak carboxylic acid groups of PAA might have a small contribution. Treatment of the as-prepared acidic hydrogel electrolyte with an alkali not only neutralized $\mathrm{HClO}_{4}$ of the hydrogel but also facilitated the conversion of polyacrylic acid to polyacrylate salt. When the alkali used was in excess, the excess alkali remained absorbed in the polymer hydrogel matrix. When this hydrogel was repeatedly washed with moderately hot millipore water, all the electrolyte species were removed and the hydrogel matrix became nonconducting. This was verified by recording the impedance spectrum of the hydrogel electrolyte with SS electrodes and the data are summarized in Table I. The as-prepared acidic PVA/PAA BHE exhibited an angled straight line in the frequency range be- 

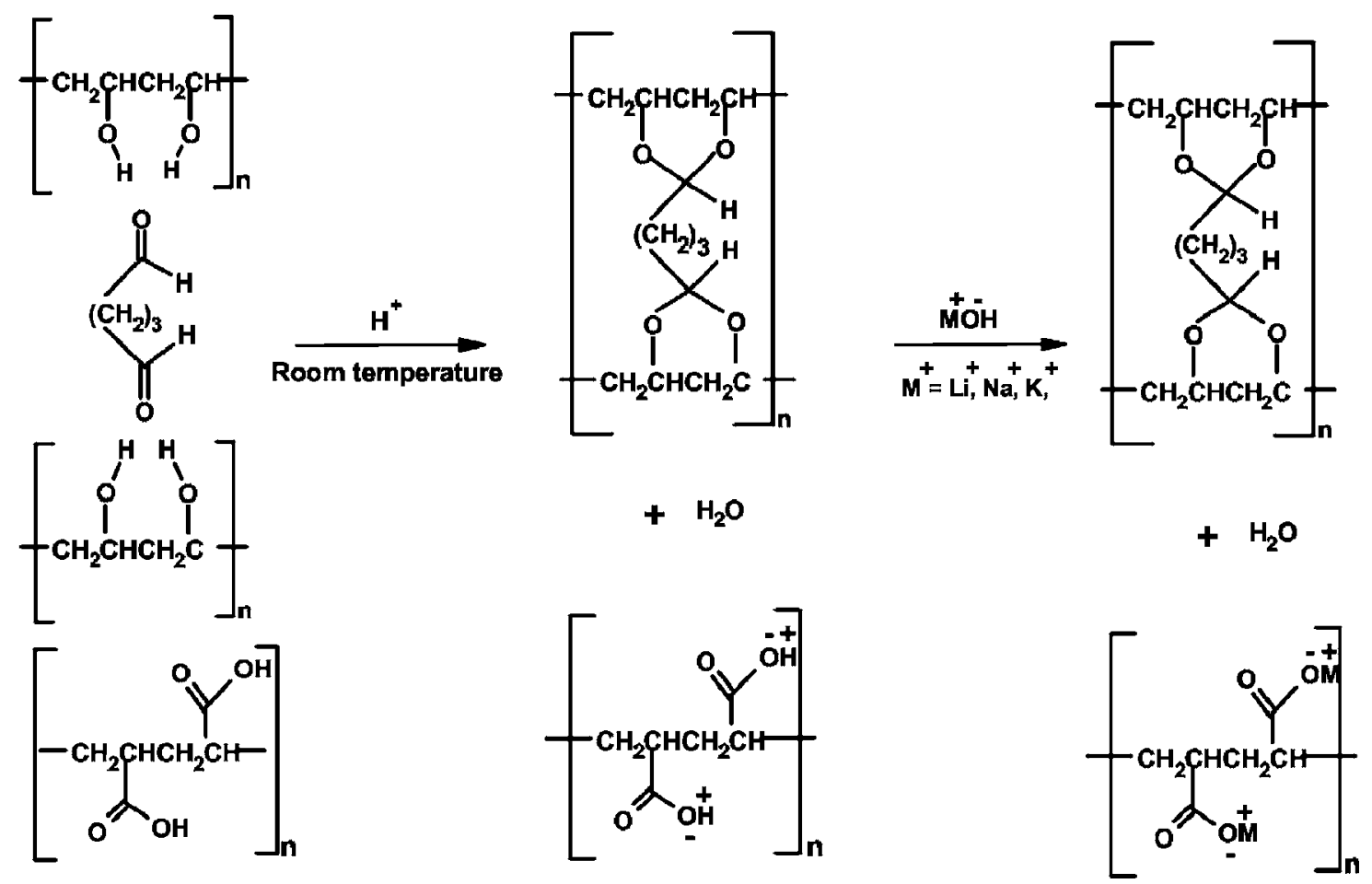

Figure 1. Reaction between glutaraldehyde and PVA blended with PAA leading to the formation of acidic cross-linked PVA/PAA blend hydrogel electrolyte (after Ref. 38) and its neutralization with alkali.

tween $200 \mathrm{~Hz}$ and $10 \mathrm{kHz}$ of the Cole-Cole plot and the bulk resistance of the same was $\sim 23 \Omega$. The polymer hydrogel obtained after neutralization of the acidic BHE with alkali and subsequent washing followed an impedance spectrum with a bulk resistance as high as $\sim 3.2 \mathrm{k} \Omega$, which indicated the absence of electrolyte in the polymer hydrogel matrix. The alkaline and neutral PVA/PAA BHEs obtained by absorbing aq. $\mathrm{NaOH}$ and aq. $\mathrm{NaCl}$ in the nonconducting polymer hydrogel matrix exhibited an angled straight line in the frequency range between $200 \mathrm{~Hz}$ and $10 \mathrm{kHz}$ of the Cole-Cole plots. The bulk resistance for the alkaline and neutral hydrogel electrolytes were $\sim 9$ and $125 \Omega$, respectively. The alkaline and neutral PVA/ PAA BHEs were transparent, pale yellow, and mechanically stable with abundant amount of water absorbed in the polymer hydrogel matrix.

Differential scanning calorimetric data for the acidic PVA/PAA BHEs as a function of polymer composition are shown in Fig. 2. It was found that with an increase in the proportion of PAA in the PVA/PAA blend, from PVA: PAA ratio of 5:0 to 1:1, the glass transition temperature $\left(T_{\mathrm{g}}\right)$ decreased from $\sim 358$ to $\sim 344 \mathrm{~K}$. This decrease in $T_{\mathrm{g}}$ of the PVA/PAA blend hydrogel in comparison to pristine PVA hydrogel might be due to the increased flexibility of the blend. ${ }^{44}$ The increased flexibility suggested by a decrease in $T_{\mathrm{g}}$ resulted in an increase in the amorphicity that, in turn, enhanced the segmental motion of the polymers in the PVA/PAA blend hydrogel as compared to the pristine PVA hydrogel. Table I. Characteristics of various PVA/PAA blend hydrogel
electrolytes.

\begin{tabular}{lcccc} 
Sl. no. & $\begin{array}{c}\text { Nature } \\
\text { of hydrogel }\end{array}$ & Electrolyte & $\begin{array}{c}\text { Bulk } \\
\text { resistance }(\Omega)\end{array}$ & Color \\
\hline 1 & Acidic & $\mathrm{HClO}_{4}$ & 23 & Colorless \\
2 & Deionized & None & 3200 & Pale yellow \\
3 & Alkaline & $\mathrm{NaOH}$ & 9 & Pale yellow \\
4 & Neutral & $\mathrm{NaCl}$ & 125 & Pale yellow
\end{tabular}

The effect of amorphicity on the ionic conductivity of nonaqueous polymer gel electrolytes with metal salts has been extensively studied in the literature. ${ }^{41}$ Decrease in $T_{\mathrm{g}}$ accompanied by an increase in amorphicity has been reported to increase the segmental motion in polymer electrolytes that, in turn, enhances the ionic conductivity. The data in Fig. 3a show that the ionic conductivity of the acidic PVA/PAA BHEs, which was of the order of $10^{-3} \mathrm{~S} \mathrm{~cm}^{-1}$, decreased with increase in the proportion of PAA in the blend. This decrease in ionic conductivity was steep in the initial stages of PAA addition (PVA/PAA ratio of 5:0 to 4:1) beyond which the decrease was small and the plot of conductivity vs volume of PAA approached a saturation value. The data in Fig. $3 \mathrm{~b}$ and c show that the

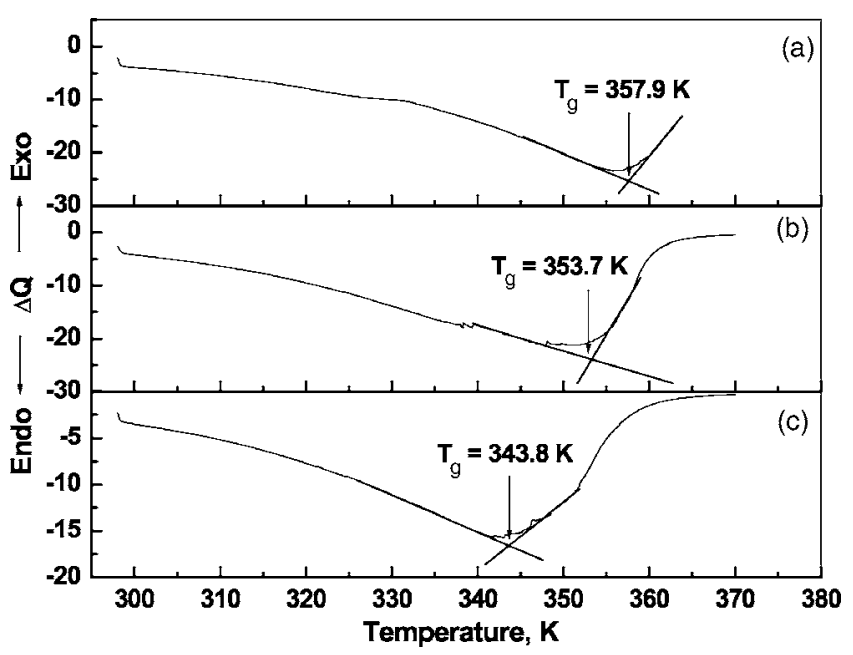

Figure 2. Differential scanning calorimetric data as a function of polymer composition for acidic PVA/PAA blend hydrogel electrolytes comprising (a) PVA: PAA $=$ 5:0, (b) PVA: PAA $=4: 1$, and (c) PVA: PAA $=1: 1$. 


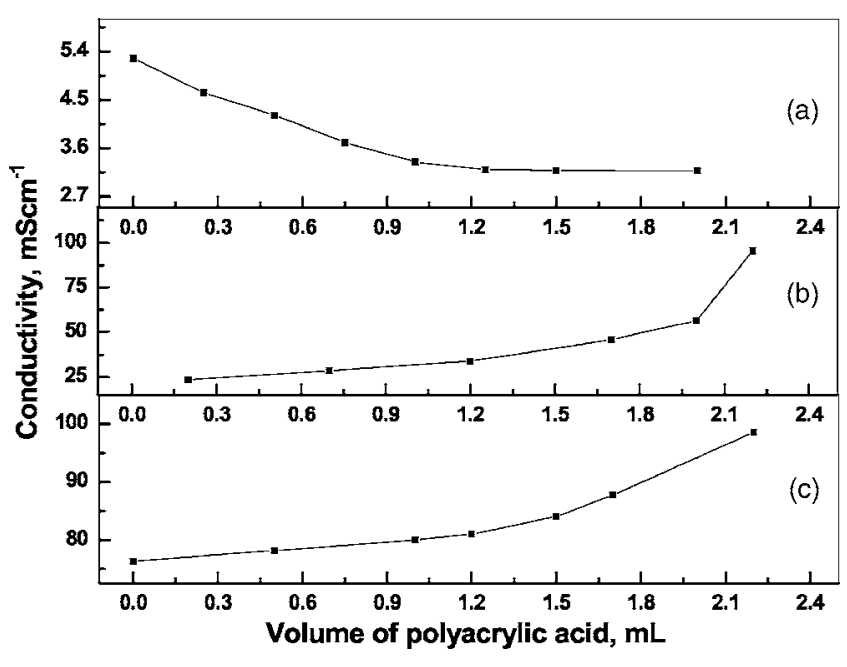

Figure 3. Ionic conductivity as a function of polymer composition for the PVA/PAA blend hydrogel electrolyte with (a) $\mathrm{HClO}_{4}$, (b) $\mathrm{NaOH}$, and (c) $\mathrm{NaCl}$ dopant.

ionic conductivity of alkaline and neutral PVA/PAA BHEs, which was of the order of $10^{-3} \mathrm{~S} \mathrm{~cm}^{-1}$, increased with increase in the proportion of PAA in the blend. This increase in ionic conductivity was slow in the initial stages of PAA addition (PVA/PAA ratio of 5:0 to $4: 1)$ beyond which the increase was fast. The response of ionic conductivity values on addition of PAA into the acidic PVA/PAA hydrogel electrolyte was contrary to that observed for the alkaline and neutral BHEs. The behavior observed in Fig. 3 is due to the combined effect of the increased flexibility in the PVA/PAA blend, as compared to the pristine PVA hydrogel, and a shift in the preferred mechanism of ionic conduction in acidic medium in contrast to the alkaline as well as neutral media. These observations could be correlated to the known behavior of this type of electrolytes. Proton conduction in the acidic PVA/PAA hydrogel electrolyte takes place by the well-established Grötthus-type mechanism, ${ }^{45,46}$ whereas ionic conduction in the alkaline as well as neutral PVA/PAA hydrogel electrolytes occurs by the usual segmental motion mechanism. ${ }^{47}$ The two mechanisms are depicted in Fig. 4a and b. As shown in Fig. 4a, the unique Grötthus-type mechanism for proton conduction in an aqueous acidic solution involves conduction of protons along one of the chains of hydrogen bonded water molecules. This mechanism explains the high conductivity of proton in aqueous acids despite its small size and large polarizability. This mechanism also explains that the proton conductivity in ice is $\sim 50$ times higher than in liquid water by taking into account the fact that hydrogen bonding is more efficient in ice (four water molecules per hydronium ion) than liquid water (three water molecules per hydronium ion). The dynamic percolation model ${ }^{47}$ developed by Druger, Nitzan, and Ratner for ion conduction in polymer electrolytes envisages ionic motion in terms of jumps between energetically equivalent neighboring positions. As shown schematically in Fig. $4 \mathrm{~b}$, a cation $\left(\mathrm{M}^{+}\right)$being a Lewis acid links with Lewis bases (B), such as oxygen atoms, associated with a polymer chain. The jump of a cation from one site to an energetically equivalent neighboring site takes place by a slow change in the local environment as a single $\mathrm{M}^{+}$-B linkage evolves with time. As the polymer gradually changes its conformation due to segmental motion, from (i) to (iv) in Fig. 4b, an accompanying translation of the cation to the neighboring site follows. The increased polymer segmental motion in PVA/PAA BHE, resulting from the increased amorphicity/disorder as the proportion of PAA in the blend was increased, brought about an increase in entropy as compared to the pristine PVA hydrogel. This increased disorder explains the decrease in proton conductivity in the acidic PVA/PAA BHE and increase in ionic conductivity in the alkaline and neutral PVA/PAA BHEs, as compared to the pristine PVA hydrogel electrolyte.

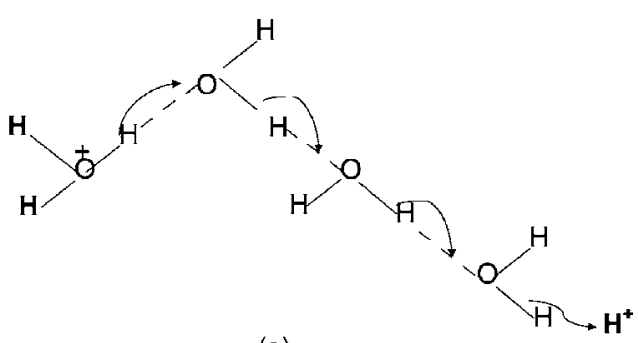

(a)

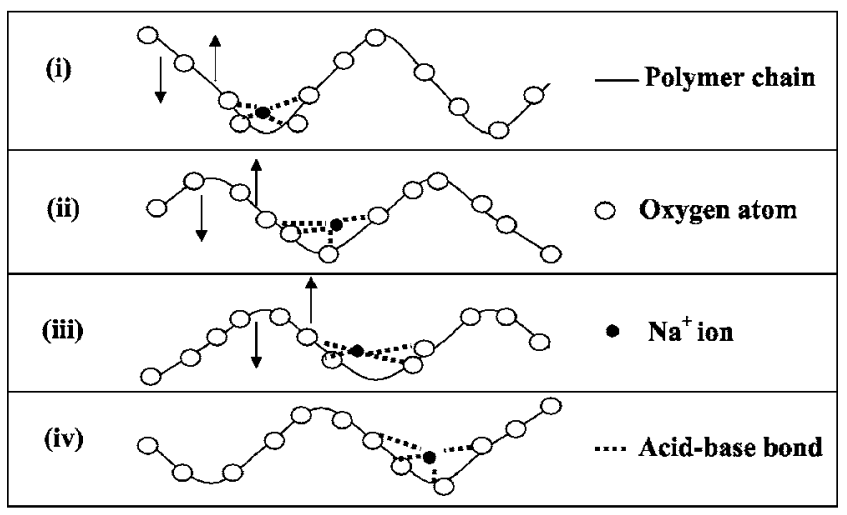

(b)

Figure 4. (a) Grötthus-type mechanism of proton conduction (after Ref. 45 and 46) in the acidic hydrogel electrolyte, and (b) segmental-motion mechanism of ionic conduction in the neutral and alkaline hydrogel electrolytes.

Ionic conduction in aqueous acidic liquids takes place by the Grötthus-type mechanism ${ }^{45,46}$ whereas segmental motion mechanism ${ }^{47}$ accounts for ionic conduction in non-acidic aqueous polymer electrolytes. The lower ionic conductivity $\left(\sim 10^{-3} \mathrm{~S} \mathrm{~cm}^{-1}\right)$ in the acidic PVA hydrogel electrolyte, as compared to that in acidic aqueous liquids $\left(10^{-1}-10^{1} \mathrm{~S} \mathrm{~cm}^{-1}\right)$, may be due to the mutually opposing effects of the Grötthus-type and segmental motion mechanisms operative in the acidic PVA hydrogel electrolyte.

The lower ionic conductivity in the acidic PVA/PAA BHEs is due to the weak acidic nature of the pendant carboxylic acid groups as the contribution from weakly acidic carboxylic acid groups in PAA to the ionic conductivity of PVA/PAA blends is negligibly small. This is evident from the fact that ionic conductivity of the acidic pristine PVA hydrogel electrolyte is comparable to that for PVA/ PAA blend hydrogel electrolytes. The conductivity rather decreased with increased proportion of PAA in the PVA/PAA blends as seen in Fig. 3a. Ionic conductivity in the acidic pristine PVA as well as blended PVA/PAA hydrogel electrolytes studied in the present investigation is understood to be mainly due to $\mathrm{HClO}_{4}$ employed to catalyze the cross-linking of PVA with glutaraldehyde. This is evident from Fig. 5a, which shows the variation of ionic conductivity of optimized acidic pristine PVA hydrogel electrolyte with varying concentration of $\mathrm{HClO}_{4}$ at room temperature. Interestingly, the ionic conductivity increased almost linearly with increase in $\mathrm{HClO}_{4}$ concentration in accordance with the Nernst-Einstein equation. ${ }^{48}$

Data in Fig. 5b show the temperature dependence of ionic conductivity of the optimized acidic pristine PVA hydrogel electrolyte. Ionic conductivity was found to increase with increase in temperature. The dependence of ionic conductivity on temperature followed the Vögel-Tamman-Fulcher (VTF) equation ${ }^{49}$

$$
\sigma(T)=A T^{-1 / 2} \exp \left[-B / k_{B}\left(T-T_{\mathrm{o}}\right)\right]
$$

In Eq. $4, A$ is a preexponential parameter proportional to the number of carrier ions, $B$ is the pseudoactivation energy related to polymer segmental motion, $k_{\mathrm{B}}$ is Boltzmann's constant, and $T_{\mathrm{o}}$ is a reference 

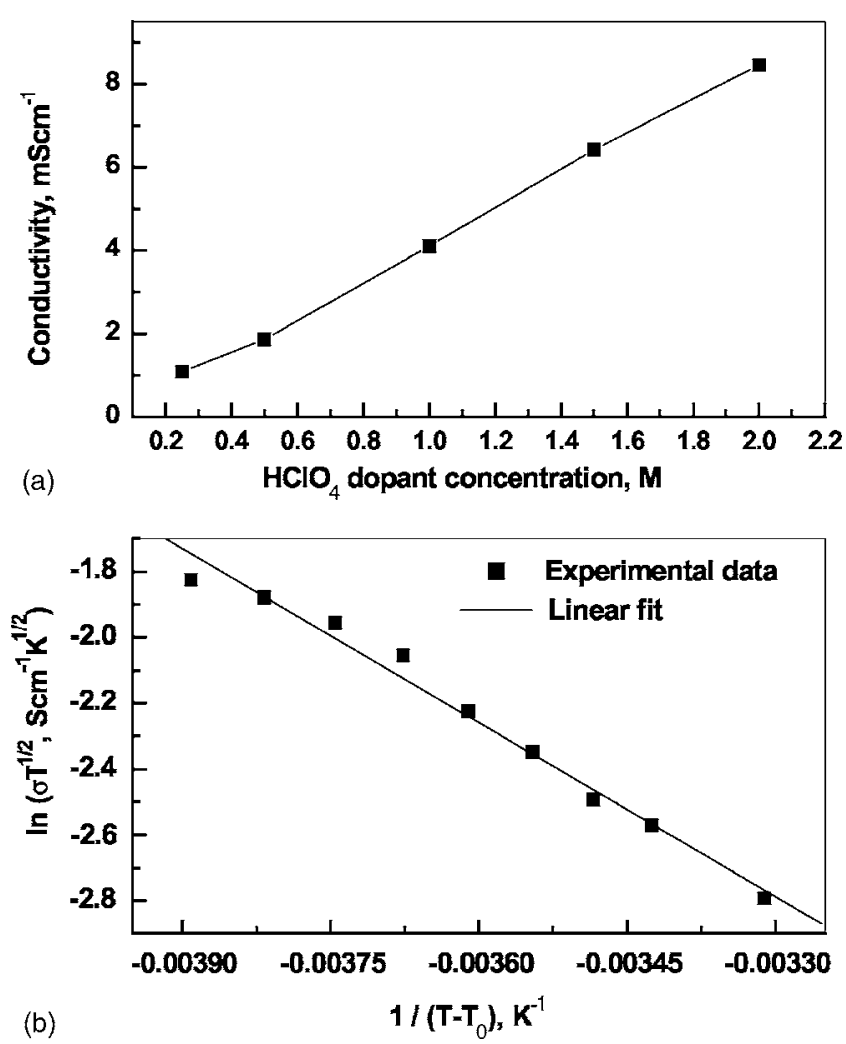

Figure 5. Variation of ionic conductivity of the acidic pristine PVA hydrogel electrolyte (a) with varying $\mathrm{HClO}_{4}$ concentration at $298 \mathrm{~K}$, and (b) with varying temperature at $1.5 \mathrm{M} \mathrm{HClO}_{4}$.

temperature at which the configurational entropy of the polymer becomes zero and is close to $T_{\mathrm{g}}$. The data on fitting to the VTF equation yielded an activation energy value of $\sim 0.15 \mathrm{eV}$ corresponding to $T_{\mathrm{o}}$ of $400 \mathrm{~K}$. The magnitude of the $R$ value for linear fitting in the VTF plot was 0.99. The near-linearity in the VTF plot shown in Fig. 5b indicates that segmental motion in PVA influences proton transport in acidic PVA hydrogel electrolytes, although the predominant mechanism happens to be the Grötthus-type mechanism as found from the discussions on Fig. 2-4.

Powder X-ray diffraction patterns of $\mathrm{RuO}_{x} \cdot \mathrm{H}_{2} \mathrm{O} / \mathrm{C}$ samples heat-treated at various temperatures for $5 \mathrm{~h}$ showed that the asprepared sample was amorphous, which transformed to crystalline form on heat treatment at $\sim 523 \mathrm{~K}$. The XRD pattern for crystalline ruthenium oxide was indexed in a tetragonal $P 4_{2} / \mathrm{mnm}$ space group. $^{40}$

Electrochemical capacitors based on cross-linked PVA/PAA BHEs with acidic, alkaline, and neutral electrolytes may be regarded as 'all solid state electrochemical capacitors.' This is because BHEs are actually solid polymer electrolytes with some water trapped in the polymer matrices during gelation. The trapped water acts as plasticizer and enhances ionic conductivity. Hydrogels are different from aqueous liquid electrolytes in that they are solid in physical state, free standing in nature, dry to touch and possess good mechanical stability. In the literature, ${ }^{42,43}$ Nafion membrane and PVA$\mathrm{KOH}$ based ECs have been referred to as all solid-state capacitors.

Cyclic voltammograms of the ECs with BP carbon electrodes and PVA/PAA BHEs with varying polymer compositions are shown in Fig. 6 (i) for acidic, (ii) for alkaline, and (iii) for neutral forms. The CVs in Fig. 6 are nearly rectangular indicating near-ideal capacitative behavior for the ECs. ${ }^{9}$ The currents in these CVs have been converted to specific capacitances as discussed in the section on Electrochemical characterization of PVA/PAA blend hydrogen electrolytes. In the case of the acidic hydrogel electrolytes, a maxi-

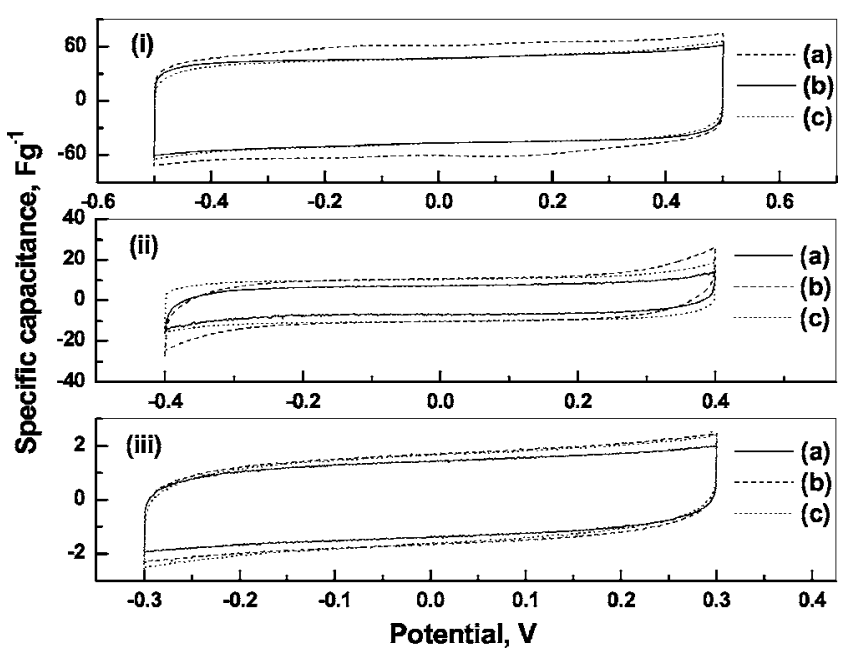

Figure 6. Cyclic voltammograms of the electrochemical capacitors with BP carbon electrodes and PVA/PAA blend hydrogel electrolytes comprising (a) PVA: PAA $=5: 0$, (b) PVA: PAA $=4: 1$, and (c) PVA: PAA = 3:2 for (i) acidic, (ii) alkaline, and (iii) neutral forms.

mum capacitance of $\sim 60 \mathrm{~F} \mathrm{~g} \mathrm{~g}^{-1}$ was observed for the EC with PVA: PAA ratio of 5:0 in the PVA/PAA blend [Fig. 6 (i, graph a)]. The capacitance decreased to $\sim 50 \mathrm{~F} \mathrm{~g} \mathrm{~g}^{-1}$ when the proportion of PAA in the blend was increased to PVA: PAA ratio of 4:1 [Fig. 6 (i, graph b)]. With further increase in the proportion of PAA in the blend to PVA: PAA ratio of 3:2, there was little decrease in the capacitance [Fig. 6 (i, graph c)]. This variation of capacitance with proportion of PAA in the blend is similar to that observed for the ionic conductivity data shown in Fig. 3a. In the case of the alkaline hydrogel electrolytes, a maximum capacitance of $\sim 10 \mathrm{~F} \mathrm{~g}^{-1}$ was observed for the EC with PVA: PAA ratio of $4: 1$ and $3: 2$ as shown in Fig. 6 (ii, graphs $\mathrm{b}$ and $\mathrm{c}$, respectively). The capacitance value decreased to $\sim 7 \mathrm{~F} \mathrm{~g}^{-1}$ when the proportion of PAA in the blend was decreased to PVA/PAA ratio of 5:0 [Fig. 6 (ii, graph a)]. It may be noted that for the alkaline hydrogel electrolyte based EC, there was an increase in capacitance with an initial increase in the proportion of PAA in the blend, following which the capacitance acquired a saturation value. Although the capacitance acquired a saturation value, the capacitative characteristics improved with increase in the proportion of PAA in the blend. This is evident from the CVs of the ECs, which tended to be both flatter and rectangular. In the case of the neutral hydrogel electrolytes, a maximum capacitance of $\sim 2 \mathrm{~F} \mathrm{~g} \mathrm{~g}^{-1}$ was observed for the ECs with PVA: PAA ratio of $4: 1$ and 3:2, respectively, as shown in Fig. 6 (iii, graphs b and c). The capacitance decreased to $\sim 1 \mathrm{~F} \mathrm{~g}^{-1}$ when the proportion of PAA in the blend was decreased to 5:0 [Fig. 6 (iii, graph a)]. It is noteworthy that, similar to the alkaline hydrogel electrolyte based ECs, the neutral hydrogel electrolyte based ECs exhibited an increase in capacitance with an initial increase in the proportion of PAA in the blend beyond which the capacitance acquired a saturation value. Although the capacitance acquired a saturation value, the capacitative characteristics improved with increase in the proportion of PAA in the blend. This further demonstrated the influence of PAA on the electrochemical characteristics of the PVA/PAA blend as reflected from its ionic conductivity measurements.

We have chosen $\mathrm{RuO}_{x} \cdot x \mathrm{H}_{2} \mathrm{O} / \mathrm{C}$ electrodes in the present investigation to emphasize that the acidic PVA/PAA BHEs may be employed with pseudocapacitative materials as well. Ruthenium oxide is one of the most widely studied pseudocapacitative materials in aqueous acidic media (liquid as well as polymeric electrolytes). It is reported to yield $\sim 720 \mathrm{~F} \mathrm{~g}^{-1}$ in aqueous $\mathrm{H}_{2} \mathrm{SO}_{4}$ solution. ${ }^{20,40} \mathrm{We}$ have employed acidic PVA/PAA BHEs in $\mathrm{RuO}_{x} \cdot \mathrm{xH}_{2} \mathrm{O} / \mathrm{C}$-based ECs to check their perfomance vis-à-vis aqueous $\mathrm{H}_{2} \mathrm{SO}_{4}$ solution. Cyclic voltammograms of the ECs with $\mathrm{RuO}_{x} \cdot x \mathrm{H}_{2} \mathrm{O} / \mathrm{C}$ electrodes and 


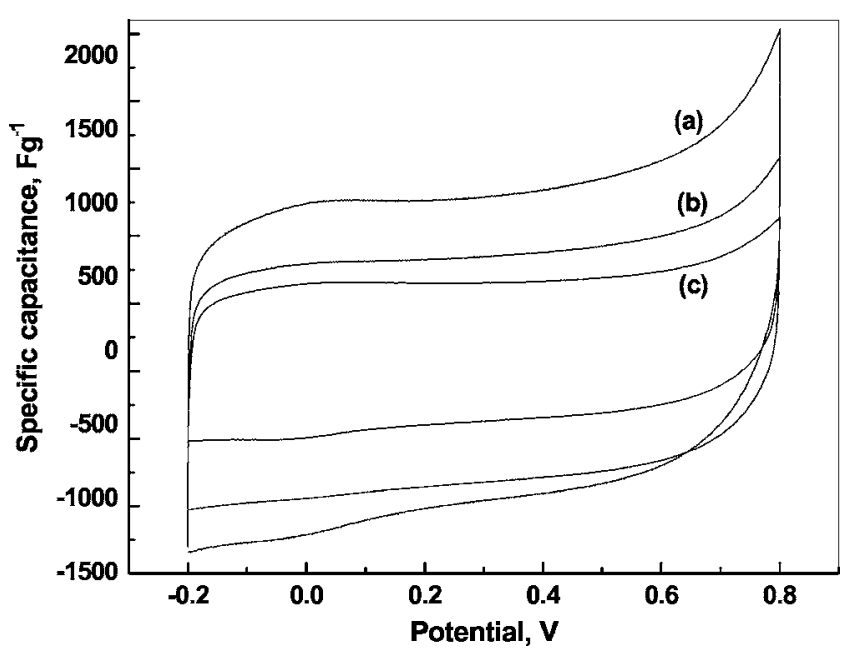

Figure 7. Cyclic voltammograms of the electrochemical capacitors with $\mathrm{RuO}_{x} \cdot x \mathrm{H}_{2} \mathrm{O} / \mathrm{C}$ electrodes and acidic PVA/PAA blend hydrogel electrolytes comprising (a) PVA: PAA $=5: 0$, (b) PVA: PAA $=4: 1$, and (c) PVA: $\mathrm{PAA}=3: 2$.

acidic PVA/PAA BHEs with varying polymer compositions are shown in Fig. 7a-c. The CVs are nearly rectangular indicating nearideal capacitative behavior for the ECs. ${ }^{9}$ Similar to the study with BP carbon electrodes, there was a decrease in capacitance with an increase in the proportion of PAA in the blend. A maximum capacitance of $\sim 1000 \mathrm{~F} \mathrm{~g}^{-1}$ was obtained for the acidic hydrogel electrolyte of composition PVA:PAA $=5: 0$. With increase in the proportion of PAA in the blend to PVA: PAA ratio of $4: 1$ and $3: 2$, the capacitance decreased to $\sim 658$ and $480 \mathrm{~F} \mathrm{~g}^{-1}$, respectively. These data suggest that the composition of the polymer blend is critical in formulating different forms of polymer hydrogel electrolytes for use in ECs. For acidic form of the PVA/PAA BHE, addition of PAA is not necessary, but the addition of PAA appears to be mandatory to evolve alkaline and neutral polymer hydrogel electrolytes of desired ionic conductivity and for their subsequent use in ECs.

Electrochemical impedance plots of the EC with $\mathrm{RuO}_{x} \cdot x \mathrm{H}_{2} \mathrm{O} / \mathrm{C}$ electrodes and acidic pristine PVA hydrogel electrolyte recorded in the frequency range between $5 \mathrm{mHz}$ and $10 \mathrm{kHz}$ with a perturbation of $5 \mathrm{mV} \mathrm{rms} \mathrm{showed} \mathrm{a} \mathrm{charge-transfer} \mathrm{resistance} \mathrm{of} \sim 32 \Omega$ and a phase angle of $\sim 82^{\circ}$. These electrochemical impedance data were analyzed in the light of the impedance data analysis due to Taberna et al. ${ }^{50}$ Plots of normalized imaginary part $|Q| /|S|$ and real part $|P| /|S|$ of the complex power $(S)$ versus frequency are shown in Fig. 8. The relaxation time constant of the EC determined from these data was found to be $\sim 0.33 \mathrm{~s}$.

Figure 9 shows cycle life data of the EC with $\mathrm{RuO}_{x} \cdot x \mathrm{H}_{2} \mathrm{O} / \mathrm{C}$ electrodes and acidic pristine PVA hydrogel electrolyte at $80 \mu \mathrm{A} \mathrm{cm}^{-2}$. The data reflects that the EC exhibited a constant pseudo-capacitance of $\sim 500 \mathrm{~F} \mathrm{~g}^{-1}$ for about 45 cycles.

\section{Conclusions}

In this study, PVA/PAA blend hydrogel electrolytes with acidic $\left(\mathrm{HClO}_{4}\right)$, alkaline $(\mathrm{NaOH})$, and neutral $(\mathrm{NaCl})$ dopants were prepared by a solution-casting method and characterized by thermal as well as electrochemical techniques. It was demonstrated that ionic conduction, in the acidic PVA/PAA hydrogel electrolyte takes place predominantly by the Grötthus-type mechanism of proton conduction, whereas that in the alkaline and neutral PVA/PAA hydrogel electrolytes takes place by the usual polymer segmental motion mechanism. Ionic conductivity of the hydrogel electrolyte was found to be of the order of $10^{-3} \mathrm{~S} \mathrm{~cm}^{-1}$ for acidic and $10^{-2} \mathrm{~S} \mathrm{~cm}^{-1}$ for alkaline and neutral forms. Temperature dependence of ionic conductivity of the acidic pristine PVA hydrogel electrolyte was found to follow the VTF equation with an activation energy of

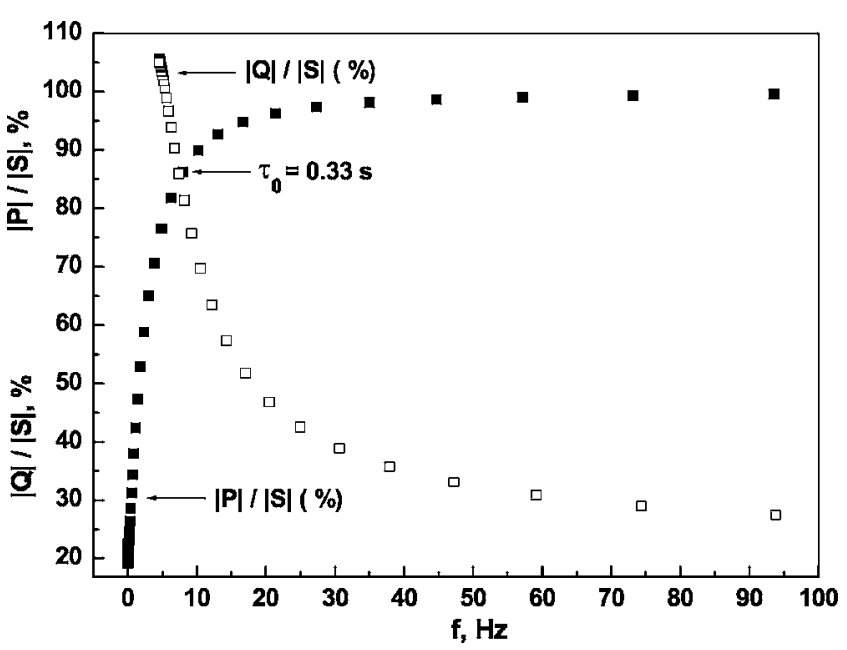

Figure 8. Plots of normalized reactive power $|Q| /|S|$ and active power $|P| /|S|$ vs frequency for the electrochemical capacitor with $\mathrm{RuO}_{x} \cdot x \mathrm{H}_{2} \mathrm{O} / \mathrm{C}$ electrodes and acidic pristine PVA hydrogel electrolyte.

$\sim 0.15 \mathrm{eV}$. The study further demonstrated that it is possible to assemble and operate all solid-state ECs with PVA/PAA BHE in acidic, alkaline, and neutral forms. For the acidic BHE-based EC, capacitance decreased with an increase in proportion of PAA in the PVA/PAA blend, whereas an opposite trend was observed for alkaline and neutral BHE-based ECs. Using acidic pristine PVA hydrogel electrolyte, a specific capacitance of $\sim 1000 \mathrm{~F} \mathrm{~g}^{-1}$ was obtained with $\mathrm{RuO}_{x} \cdot x \mathrm{H}_{2} \mathrm{O} / \mathrm{C}$ electrodes as opposed to the value of $\sim 60 \mathrm{~F} \mathrm{~g}^{-1}$ obtained with $\mathrm{BP}$ carbon electrodes. The relaxation time constant of the EC with $\mathrm{RuO}_{x} \cdot x \mathrm{H}_{2} \mathrm{O} / \mathrm{C}$ electrodes and acidic pristine PVA hydrogel electrolyte was found to be $\sim 0.33 \mathrm{~s}$. The EC exhibited a stable pseudocapacitance of $\sim 500 \mathrm{~F} \mathrm{~g}^{-1}$ for about 45 chargedischarge cycles.

\section{Acknowledgments}

N.A.C. is grateful to the Council of Scientific and Industrial Research, New Delhi for giving him a Research Fellowship.

The Indian Institute of Science assisted in meeting the publication costs of this article.
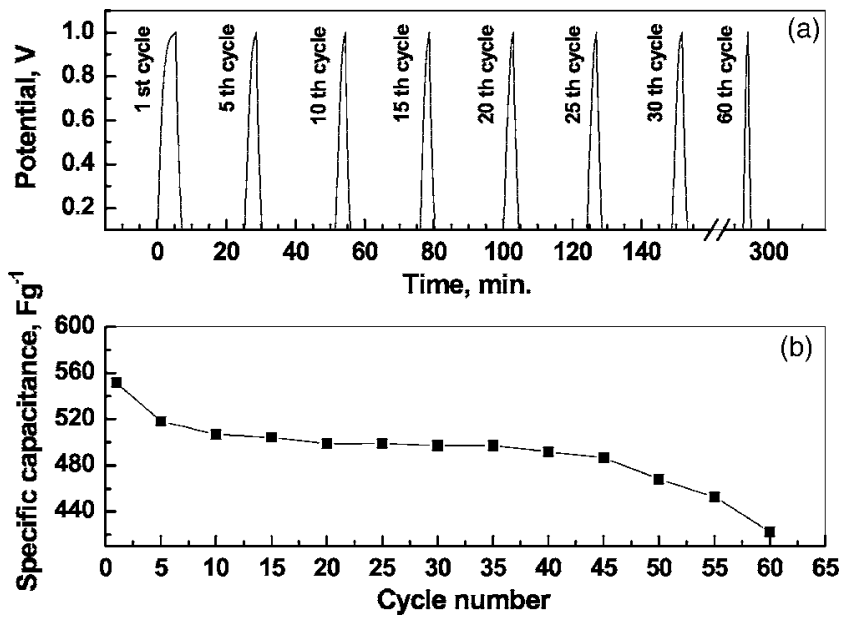

Figure 9. (a) Galvanostatic charge-discharge plots for various chargedischarge cycles, and (b) cycle life data of the electrochemical capacitor with $\mathrm{RuO}_{x} \cdot x \mathrm{H}_{2} \mathrm{O} / \mathrm{C}$ electrodes and acidic pristine PVA hydrogel electrolyte recorded at $80 \mu \mathrm{A} \mathrm{cm}^{-1}$. 


\section{References}

1. Polymer Electrolyte Review J. R. MacCallum and C. A. Vincent, Editors, Vol. 1/2, Elsevier, London (1987-89).

2. F. M. Gray, Solid Polymer Electrolytes-Fundamentals and Technological Applications, VCH, Weinheim (1991).

3. B. Scrosati, Application of Electroactive Polymers, Chapman and Hall, London (1993).

4. K. M. Abraham and M. Alamgir, J. Electrochem. Soc, 137, 1657 (1990).

5. F. B. Dias, L. Plomp, and J. B. J. Veldhuis, J. Power Sources, 88, 169 (2000).

6. Y. Matsuda, K. Inoue, H. Takeuchi, and Y. Okuhama, Solid State Ionics, 113-115, 103 (1998).

7. M. Ishikawa, M. Ihara, M. Morita, and Y. Matsuda, Electrochim. Acta, 40, 2217 (1995).

8. S. Mitra, A. K. Shukla, and S. Sampath, J. Power Sources, 101, 213 (2001).

9. B. E. Conway, Electrochemical Supercapacitors: Scientific Fundamentals and Technological Applications, Kluwer Academic Publishers/Plenum Press, New York (1999).

10. E. Frackowiak and F. Béguin, Carbon, 39, 937 (2001)

11. J. Gamby, P. L. Taberna, P. Simon, J. F. Fauvarque, and M. Chesneau, J. Power Sources, 101, 109 (2001).

12. S. T. Mayer, R. W. Pekala, and J. L. Kaschmitter, J. Electrochem. Soc., 140, 446 (1993).

13. R. W. Pekala, J. C. Farmer, C. T. Alviso, T. D. Tran, S. T. Mayer, J. M. Miller, and B. Dunn, J. Non-Cryst. Solids, 225, 74 (1998).

14. J. Wang, S. Q. Zhang, Y. Z. Guo, J. Shen, S. M. Attia, B. Zhou, G. Z. Zeng, and Y. S. Gui, J. Electrochem. Soc., 148, D75 (2001).

15. E. Frackowiak, K. Meternier, V. Bertagna, and F. Béguin, Appl. Phys. Lett., 77, 2421 (2000).

16. E. Frackowiak, K. Jurewicz, S. Delpeux, and F. Béguin, J. Power Sources, 97-98, 822 (2001).

17. M. Mastragostino, C. Arbizzani, and F. Soavi, Solid State Ionics, 148, 493 (2002).

18. B. E. Conway, J. Electrochem. Soc., 138, 1539 (1991).

19. S. Sarangapani, B. V. Tilak, and C. P. Chen, J. Electrochem. Soc., 143, 3791 (1996).

20. J. P. Zheng, P. J. Cygan, and T. R. Jow, J. Electrochem. Soc., 142, 2699 (1995).

21. V. Srinivasan and J. W. Weidner, J. Electrochem. Soc., 147, 880 (2000).

22. T. Kudo, Y. Ikeda, T. Watanabe, M. Hibino, M. Miyayama, H. Abe, and K. Kajita, Solid State Ionics, 152-153, 833 (2002).

23. Y. U. Jeong and A. Manthiram, Electrochem. Solid-State Lett., 3, 205 (2000).
24. A. Yamada and J. B. Goodenough, J. Electrochem. Soc., 145, 737 (1998).

25. H. Y. Lee and J. B. Goodenough, J. Solid State Chem., 144, 220 (1999).

26. H. P. Stadniychuk, M. A. Anderson, and T. W. Chapman, J. Electrochem. Soc., 143, 1629 (1996).

27. J. J. Xu, A. J. Kinser, B. B. Owens, and W. H. Smyrl, Electrochem. Solid-State Lett., 1, 1 (1998)

28. P. N. Gupta and K. P. Singh, Solid State Ionics, 86-88, 319 (1996).

29. W. Yong-gang and Z. Xiao-gang, Electrochim. Acta, 49, 1957 (2004).

30. S. Guinot, E. Salmon, J. F. Penneau, and J. F. Fauvarque, Electrochim. Acta, 43 1163 (1998).

31. N. Vassal, E. Salmon, and J. F. Fauvarque, J. Electrochem. Soc., 146, 20 (1999).

32. C-C. Yang, J. Power Sources, 109, 22 (2002).

33. C. Iwakura, S. Nohara, N. Furukawa, and H. Inoue, Solid State Ionics, 148, 487 (2002).

34. C. Iwakura, H. Wada, S. Nohara, N. Furukawa, H. Inoue, and M. Morita, Electrochem. Solid-State Lett., 6, A37 (2003).

35. C. Louis, J. F. Chailan, P. Bartolomeo, and J. L. Vernet, Polymer, 42, 7107 (2001).

36. D. R. Paul and S. Newman, Polymer Blends, Vol. I, Academic Press, New York (1978).

37. L. A. Utracki, Polymer Alloys and Blends, Hanser, New York (1989).

38. C. O. Dasenbrock, T. H. Ridgway, C. J. Seliskar, and W. R. Heineman, Electrochim. Acta, 43, 3497 (1998).

39. W. H. Philipp and W. K. Street, Jr., U.S. Pat. 5,371,110 (1994).

40. J. P. Zheng and T. R. Jow, J. Electrochem. Soc., 142, L6 (1995).

41. P-L. Kuo,W-J. Liang, and T-Y. Chen, Polymer, 44, 2957 (2003).

42. P. Staiti, M. Minutoli, and F. Lufrano, Electrochim. Acta, 47, 2795 (2002).

43. C.-C. Yang, S.-T. Hsu, and W.-C. Chien, J. Power Sources, In press.

44. B. Serrano, I. F. Pierola, J. Bravo, and J. Baselga, J. Mater. Process. Technol., 141, 123 (2003).

45. A. Nowak, in Proton Conductors, Ph. Colomban, Editor, Cambridge University Press, Cambridge (1992).

46. O. F. Mohammad, D. Pines, J. Dreyer, E. Pines, and E. T. J. Nibbering, Science, 310, 83 (2005)

47. M. A. Ratner and D. F. Shriver, Chem Rev (Washington, D.C.), 88, 109 (1988)

48. A. R. West, Solid State Chemistry and Its Applications, p. 462, John Wiley \& Sons, New York (1987)

49. R. Baskaran, S. Selvasekarapandian, N. Kuwata, J. Kawamura, and T. Hattori, Materials Chemistry and Physics, In press.

50. P. L. Taberna, P. Simon, and J. F. Fauvarque, J. Electrochem. Soc., 150, A292 (2003). 